\title{
Predicción y análisis de emisiones de mezclas combustibles diésel/biodiésel modificadas con nanopartículas de alúmina y ácido oleico
}

\section{Prediction and analysis of emissions from diesel/biodiesel fuel blends modified with alumina nanoparticles and oleic acid}

\section{Previsão e análise das emissões de mistura de combustíveis diesel/biodiesel modificadas com nanopartículas de alumina e ácido oleico}

\author{
Andrés D. Peñaloza; Andy J. Rincón; Adriana P. Herrera; Karina A. Ojeda* \\ Programa de Ingeniería Química. Facultad de Ingeniería. Universidad de Cartagena, Campus Piedra de Bolívar. \\ Avenida del Consulado Calle 30 \# 48-152. Código Postal 130015, Cartagena, Colombia. \\ *kojedad@unicartagena.edu.co
}

Fecha Recepción: 01 de agosto de 2016 Fecha Aceptación: 17 de abril de 2017

\begin{abstract}
Resumen
El software Diésel-RK fue utilizado para evaluar el efecto del uso de un aditivo basado en nanopartículas de alúmina y ácido oleico para la reducción de emisiones de gases contaminantes en mezclas diésel/biodiésel. Para esto, se estimaron las propiedades físico-químicas de las mezclas combustibles con ayuda de correlaciones para dispersiones coloidales y reglas de mezclado, fijando concentraciones de 10 y $20 \mathrm{ppm}$ del aditivo. Los resultados obtenidos mostraron reducciones del $11 \%$ y $25 \%$ en emisiones de $\mathrm{SO}_{2}$ y material particulado, respectivamente; mientras que las emisiones de $\mathrm{CO}_{2}$ no mostraron mejoras significativas, así como tampoco se presentaron mejoras en el consumo específico de combustible, los cuales permanecieron por debajo del 1,0\%. En cuanto a las emisiones de $\mathrm{NO}_{2}$, se observó un incremento desde $2,1 \mathrm{~g} / \mathrm{kWh}$ a $3,1 \mathrm{~g} / \mathrm{kWh}$ con la adición de las diversas concentraciones de nanoaditivo; de esta forma se evidenciaron mejoras en la disminución en el retraso de los tiempos de ignición, lo cual es apropiado para el adecuado funcionamiento de los motores.
\end{abstract}

Palabras clave: nanoaditivos, biocombustibles, diésel, gases contaminantes.

\begin{abstract}
Diesel-RK software was used to evaluate the effect of alumina nanoparticles and oleic acid as additives for reducing greenhouse gas emissions in diesel/biodiesel fuel blends. Thus, it was estimated the physicochemical properties of the fuel blends using correlations for colloidal dispersions and mixing rules. This was achieved by fixing the concentrations of the additives at 10 and 20ppm. The results showed reductions of $11 \%$ and $25 \%$ for $\mathrm{SO}_{2}$ emissions and particulate material, respectively; while $\mathrm{CO}_{2}$ emissions showed no significant improvements, as well as no significant enhancement was estimated for specific fuel consumption, which remained below $1.0 \%$. Regarding $\mathrm{NO}_{2}$ emissions, an increase from $2.1 \mathrm{~g} / \mathrm{kWh}$ to $3.1 \mathrm{~g} / \mathrm{kWh}$ was observed for the addition of several concentration of nanoadditives; thus, it was evidenced a decrease in the lag time of ignition, which is appropriate for the proper operation of engines.
\end{abstract}

Keywords: nanoadditives, biofuel, diesel, greenhouse gas.

Cita: Peñaloza AD, Rincón AJ, Herrera AP, Ojeda KA. Predicción y análisis de emisiones de mezclas combustibles diesel/biodiesel modificadas con nanopartículas de alúmina y ácido oleico. rev.ion. 2017;30(1):45-55. 


\section{Resumo}

O software Diesel-RK foi usado para avaliar o efeito da utilização de um aditivo baseado em nanopartículas de alumina e ácido oleico para reduzir as emissões de gases poluentes nas misturas diesel/biodiesel. Para isso, foram estimadas as propriedades físico-químicas das misturas de combustíveis usando correlações para dispersões coloidais e regras de mistura, com concentrações fixas de 10 e 20ppm do aditivo. Os resultados revelaram reduções significativas de $11 \%$ e $25 \%$ das emissões $\mathrm{SO}_{2}$ e do material particulado, respectivamente; enquanto as emissões de $\mathrm{CO}_{2}$ não mostraram nenhuma melhora importante nas emissões, nem mostrou melhorias no consumo específico de combustível, que se manteve inferior a $1,0 \%$. Agora, respeito as emissões de $\mathrm{NO}_{2}$, observou-se um aumento de $2,1 \mathrm{~g} / \mathrm{kWh}$ para 3,1g/ kWh, com a adição de várias variantes do nano-aditivo; assim, constatou-se uma diminuição do tempo de retardamento da ignição, o que é apropriado para o bom funcionamento dos motores.

Palabras-chave: nanoaditivos, biocombustivel, diesel, gases de efeito estufa.

\section{Introducción}

En la última década, las investigaciones sobre contaminantes atmosféricos se han orientado a la evaluación de sus efectos sobre la salud humana y que estrategias podrían implementarse para disminuir su concentración en el ambiente [1]. En este sentido, muchos estudios relacionan el incremento de casos de enfermedades respiratorias agudas con el aumento en la concentración de gases contaminantes como dióxido de azufre $\mathrm{SO}_{2}$, óxidos de nitrógeno $\mathrm{NOx}$, dióxido de carbono $\mathrm{CO}_{2}$, monóxido de carbono $\mathrm{CO}$ y material particulado provenientes en gran medida de la quema de combustibles fósiles $[1,2]$.

Los biocombustibles líquidos han sido propuestos como una alternativa al uso de combustibles fósiles, al tiempo que se logra una reducción de emisiones de contaminantes; sin embargo, sus proporciones en mezclas aún son muy bajas (menores al 10\%) por lo que todavía generan grandes cantidades efectos ambientales $[1,3,4]$. En respuesta a la demanda de combustibles de mayor calidad se ha evaluado el efecto de incorporar materiales aditivos (metales y sustancias orgánicas) con el propósito de mejorar las propiedades físico-químicas de las mezclas de combustibles diésel/biodiésel $[3,4]$

Entre los materiales evaluados como potenciales aditivos de mezclas combustibles se destacan los sintetizados en la escala nanométrica, los cuales presentan como ventajas la presencia de una mayor cantidad de sitios activos, lo cual los hace ver como catalizadores más eficientes capaces de asistir en los procesos de oxidación de los combustibles durante la combustión sin posibilidad de generar obstrucciones en los sistemas de filtros e inyección en motores (caso de aditivos en el orden de micras), además de reducir el desgaste de las partes móviles del motor $[3,5]$.

Entre las nanopartículas estudiadas se encuentran el óxido de cerio [1], óxido de cobalto y aleaciones de magnesio-aluminio [6], como también las nanopartículas de alúmina $\left(\mathrm{Al}_{2} \mathrm{O}_{3}\right)$ las cuales pueden obtenerse a través de síntesis química de bajo costo, ofreciendo mejoras en la reducción de emisiones de gases contaminantes y rendimiento en la combustión [4]. Ganesh y Gowrishankar estudiaron la aplicación de nanopartículas de óxido de cobalto como aditivos para la combustión de hidrocarburos, observando una reducción significativa en las emisiones de NOx, atribuida a la descomposición del monóxido de nitrógeno (NO) en la superficie reactiva del óxido de cobalto [6].

En otros estudios, Jones et al. investigaron el efecto de las nanopartículas de alúmina en la mejora de propiedades del bioetanol, observando un incremento en la eficiencia de combustión. En adición, estimaron a partir de modelos de equilibrio termodinámico incrementos considerables en la temperatura de flama adiabática de hasta un $8,27 \%$ con respecto al bioetanol puro para concentraciones relativamente bajas de las nanopartículas de alúmina de alrededor del $10 \%$ v [4]. Las investigaciones en el uso de nanopartículas de alúmina como aditivos combustibles se han enfocado principalmente en el efecto de la concentración de nanopartículas sobre las propiedades del biocombustibles; pero la evaluación de emisiones aún es limitada [4]. Teniendo en cuenta que el uso de nanoaditivos representa una alternativa de mejora en la industria de biocombustibles y sus mezclas, en este trabajo se evaluó el efecto del uso de nanopartículas de alúmina y ácido oleico para la reducción de emisiones de gases contaminantes y material particulado de una mezcla de combustible diésel/biodiésel usando el software Diésel-RK considerando el uso de biodiésel comercial B4 (96\%v de diésel / 4\%v de biodiésel) con nanoaditivos en concentraciones de 10 y $20 \mathrm{ppm}$. 


\section{Metodología}

El desarrollo de la investigación se estructuró en las siguientes etapas: (i) caracterización del combustible sin aditivo, para lo cual se escogió la mezcla biodiésel B4 debido a la disponibilidad de un importante número de propiedades fisicoquímicas; (ii) estimación de las propiedades del nanoaditivo (suspensión coloidal de nanopartículas de alúmina en ácido oleico) con ayuda de correlaciones para dispersiones coloidales diluidas reportadas en literatura [7-9]; (iii) aplicación de reglas de mezclado para estimación de las propiedades del combustible B4 con los nanoaditivos en concentraciones de 10 y $20 \mathrm{ppm}$ (estos valores fueron seleccionados de acuerdo a lo reportado en la literatura referente a aplicaciones de nanoaditivos en mezclas combustibles $[1,3,5])$; y (iv) evaluación de emisiones mediante el software Diésel-RK considerando las especificaciones del motor de estudio junto con las propiedades estimadas de cada una de las mezclas de combustibles y nanoaditivos.

Las especificaciones del biodiésel B4 fueron suministradas por la Coordinación de Inspección de Calidad de Ecopetrol S.A. En adición, se usaron correlaciones experimentales para estimar otras propiedades, tales como Capacidad Calorífica (Cp) [10], Poder Calorífico Alto (HHV) [11] y Calor de Vaporización ( $\Delta$ Hvap) [12] (Tabla 1$)$.

Tabla 1. Correlaciones usadas para la estimación de propiedades.

\begin{tabular}{|c|c|}
\hline Propiedad estimada & Correlación \\
\hline $\begin{array}{c}\text { Valor Calorífico Alto, } \\
\qquad H H V\end{array}$ & $\begin{array}{l}\qquad H H V=0,4527 v-0,0008 \rho-0,0003 F P+40,3667,(\mathrm{MJ} / \mathrm{kg}) \\
\text { Donde } v \text { es la viscosidad cinemática }(\mathrm{cSt}), \text { es la densidad }(\mathrm{g} / \mathrm{L}) \text { y } F P \text {, el punto de } \\
\text { inflamación }(K) \text { de la mezcla }\end{array}$ \\
\hline $\begin{array}{l}\text { Calor de vaporización, } \\
\qquad \Delta \mathrm{H}_{v a p}\end{array}$ & $\begin{array}{c}\Delta \mathrm{H}_{\text {vap }}=A 1+A 2,(\mathrm{~kJ} / \mathrm{mol}) \\
A_{1}=15,1845-0,922 \ln |\ln | \frac{-61,311+S^{-0,03768419 T_{b}+1}}{S}|| \\
A_{2}=0,000203975 T_{b}\left(\frac{-21,212111}{-10,969+41,0027905 S^{56,27}}\right)-0,33135 \ln \left|75,2754-S^{-0,039595 T_{b}}\right|\end{array}$ \\
\hline $\begin{array}{l}\text { Capacidad calorífica, } \\
\qquad P p\end{array}$ & $\begin{array}{l}\text { Donde } S \text { es la gravedad específica y } T_{b} \text { es la temperatura normal de ebullición }(K) \text { de la } \\
\text { mezcla } \\
\qquad C p=24,5\left(a_{11} \alpha+a_{12} \alpha^{2}\right)+\left(a_{21} \alpha+a_{22} \alpha^{2}\right) T+\left(a_{31} \alpha+a_{32} \alpha^{2}\right) T^{2},(J / g K) \\
\qquad \alpha=\frac{\sum_{i=1}^{n} \omega_{i} / M_{i}}{\sum_{i=1}^{n} \omega_{i}} \\
\text { Donde } T \text { es la temperatura }(K), \omega_{i} \text { y } M_{i} \text {, es la fracción másica y peso molecular del } \\
\text { elemento } i \text { en la mezcla, respectivamente. Mientras que } a_{i j} \text { son constantes calculadas } \\
\text { por los autores. }\end{array}$ \\
\hline
\end{tabular}

Para la preparación del nanoaditivo (suspensión coloidal de nanopartículas de alúmina en ácido oleico) se consideró el uso de nanopartículas de alúmina sintetizadas por el método de sol-gel y calcinadas a $1000^{\circ} \mathrm{C}$, las cuales exhiben una fase cristalina $\alpha \mathrm{Al}_{2} \mathrm{O}_{3}$ con un tamaño cristalino promedio de $280 \mathrm{~nm}$, de acuerdo a trabajos previos reportados por nuestro grupo de investigación [13]. La concentración de nanopartículas de alúmina en ácido oleico se fijó en $16 \mathrm{mg} / 100 \mathrm{~mL}$ (equivalente a $0,004 \% \mathrm{v}$, teniendoencuentauna densidad de $4 \mathrm{~g} / \mathrm{mL}$ para las nanopartículas de alúmina [13]). El efecto de las nanopartículas en las propiedades del ácido oleico se estimó mediante el uso de correlaciones 
para dispersiones coloidales diluidas, a partir de las cuales se determinaron diversos parámetros, tales como densidad (Ecuación 1) [7], viscosidad dinámica (Ecuación 2) [8], capacidad calorífica (Ecuación 3) [7] y masa molecular (Ecuación 4) [9]. En estas ecuaciones, los subíndices $n f, b$ y $s$, representan las propiedades del nanoaditivo, del ácido oleico y de las nanopartículas de alúmina, respectivamente; $\varnothing$ es la fracción volumétrica y $\varphi$ representa el porcentaje volumétrico de las nanopartículas en el ácido oleico.

$$
\begin{gathered}
\rho_{n f}=\left(1-\phi_{s}\right) \rho_{b}+\phi_{s} \rho_{s} \\
\mu_{n f}=\left(1+7,3 \varphi+123 \varphi^{2}\right) \mu_{b} \\
C_{p, n f}=\frac{\left(1-\phi_{s}\right) \rho_{b} C_{p, b}+\phi_{s} \rho_{s} C_{p, s}}{\rho_{n f}} \\
M_{n f}=\left(1-\phi_{s}\right) M_{b}+\phi_{s} M_{s}
\end{gathered}
$$

No se tuvo en cuenta la modificación de las propiedades de calor de vaporización y poder calorífico del ácido oleico por la adicción de las nanopartículas de alúmina, debido a que este nanomaterial no reacciona durante el proceso de combustión [7,11], por lo que se sugiere que no hay contribución de la alúmina en el calor liberado durante la reacción. En cuanto a la tensión superficial, los resultados reportados por Tanvir y Quiao para nanopartículas de $\mathrm{Al}$ y $\mathrm{Al}_{2} \mathrm{O}_{3}$ en n-decano, muestran que a concentraciones muy bajas ( $<3-4 \%$ en peso), hay poca influencia en esta propiedad debido a que la distancia entre partículas es lo suficientemente grande para una dispersión coloidal diluida [14].

Luego de definir todas las propiedades requeridas para la mezcla B4 y la suspensión base (ácidooleico/ nanopartículas), se estimaron las propiedades de la mezcla combustible(B4/ácidooleico/nanopartículas) empleando las reglas de mezclado básicas de Kay y Grunberg-Nissan [15]. La expresión genérica de la regla de mezclado de Kay es:

$$
\varphi_{m}=\sum_{i}^{n} x_{i} \varphi_{i}
$$

Donde, $\varphi_{m}$ es la propiedad estimada de la mezcla, $x_{i}$ representa la fracción (másica, molar o volumétrica) del componente $i$ en la mezcla y $\varphi_{i}$ corresponde a la propiedad individual del componente $i$. La expresión de la regla de mezclado de GrunbergNissan, empleada únicamente para la viscosidad, está dada por [15]:

$$
\ln \left(\mu_{m}\right)=\sum_{i}^{n} x_{i} \ln \left(\mu_{i}\right)+\sum_{i}^{n} \sum_{j}^{n} x_{i} x_{j} G_{i, j}
$$

Donde, $\mu_{m}$ es la viscosidad estimada de la mezcla, $x_{i}\left(\mathrm{o} \quad x_{j}\right)$ representa la fracción del componente $i$ (o $j$ ) en la mezcla; $\mu_{i}$ la viscosidad del componente $i$ y $G_{i, j}$ corresponde al parámetro de interacción entre componente $i$ y $j$. El empleo de estas reglas de mezclado se sustenta en la similitud en la estructura química, no polaridad y completa miscibilidad de los componentes en la mezcla, por lo que se esperaría no existiesen interacciones entre estos [15]. Además de los bajos valores de desviación reportados por Benjumea y colaboradores, con los cuales sugieren la viabilidad de estas reglas en la predicción de propiedades básicas de mezclas biodiésel-diésel. El empleo de una base de cálculo másica, volumétrica o molar en las reglas de mezclado anteriores está ligado al modo como fueron desarrolladas para cada propiedad en los estudios y documentos consultados $[15,16]$.

Una vez caracterizadas todas las propiedades de las mezclas a evaluar $(0,10$ y $20 \mathrm{ppm})$, se evaluó el desempeño teórico en el proceso de combustión mediante simulación, para lo cual se consideraron las características del motor para un auto Renault Logan $85 \mathrm{hp} 1,5 \mathrm{dCi}$, generando reportes de emisiones para cada mezcla combustibles $(0,10$ y $20 \mathrm{ppm})$ a cada una de las revoluciones evaluadas: 2000-3000rpm, con cambios de 250rpm. Esta información fue estudiada y se evaluó el cambio en emisiones de gases contaminantes: $\mathrm{CO}_{2}, \mathrm{SO}_{2}, \mathrm{NO}_{x}$ y material particulado por unidad de energía liberada y su posible relación con las composiciones de las mezclas combustibles.

\section{Resultados y Análisis}

\section{Estimación de propiedades para las mezclas}

Para determinar el efecto de los nanoaditivos en la mezcla de combustible, se determinaron las propiedades de la suspensión base de ácido oleico y nanopartículas de alúmina. Un resumen de las propiedades estimadas se muestra en la Tabla 2, las cuales se calcularon tomando como base el porcentaje de suspensión de $0,004 \%$ v de las nanopartículas en el ácido oleico. 
Tabla 2. Efecto de nanopartículas de alúmina en la suspensión base de ácido oleico.

\begin{tabular}{ccccc}
\hline Parámetro & Unidad & Ácido oleico & $\mathbf{n}-\mathrm{Al}_{2} \mathbf{O}_{3}$ & Á.oleico+ $\mathbf{n}-\mathrm{Al}_{2} \mathbf{O}_{3}$ \\
\hline Densidad a $15^{\circ} \mathrm{C}$ & $\mathrm{kg} / \mathrm{m}^{3}$ & 895 & 3880 & 895,12 \\
Viscosidad cinemática a & $\mathrm{cSt}$ & 27,93 & ---- & 28,79 \\
$40^{\circ} \mathrm{C}$ & $\mathrm{J} / \mathrm{kg} \mathrm{K}$ & 1742,37 & 729 & 1742,19 \\
Capacidad calorífica & $\mathrm{kg} / \mathrm{kgmol}$ & 282,47 & 101 & 282,49 \\
Masa molecular & &
\end{tabular}

Se pudo observar que el efecto de las nanopartículas de alúmina en las propiedades del ácido oleico (densidad y capacidad calorífica) fue despreciable, debido a la muy baja concentración volumétrica de las nanopartículas en la suspensión. En cuanto a la viscosidad, se observó un aumento de esta propiedad en la suspensión base con la adición de las nanopartículas de alúmina, similar a las mejoras reportadas para nanopartículas de $\mathrm{CeO}_{2}$ en biodiésel de Jatropha [5]. La viscosidad es una propiedad clave en la formulación de combustibles, por lo que cualquier aumento adecuado de esta con el empleo de aditivos darán lugar a mejoras considerables en el desempeño global de los motores desde su respuesta hasta sus perfiles de emisiones.

Las propiedades de las mezclas de nanoaditivos con combustibles se determinaron a partir de las reglas de mezclado y las ecuaciones (5) y (6). La Tabla 3 muestra las propiedades estimadas para las muestras de combustibles con y sin adición de las nanopartículas. Además, se presentan las especificaciones de calidad del combustible diésel corriente y sus mezclas con biocombustibles acorde a la resolución 182087 de 17 de diciembre de 2007, fijada por el Ministerio de Ambiente,
Vivienda y Desarrollo Territorial, y Ministerio de Minas y Energía de Colombia.

A partir de la información presentada en la Tabla 3 , se puede observar que las propiedades de las mezclas combustibles permanecen dentro de las especificaciones requeridas en el país tras el empleo deladitivo, destacando mejoras importantes en sus características como reducciones en el contenido de azufre, y mejoras en la viscosidad para las mezclas B4-10ppm y B4-20ppm. Esto se debe a que la suspensión base de ácido oleico con nanopartículas de alúmina $\left(\mathrm{n} \cdot \mathrm{Al}_{2} \mathrm{O}_{3}\right)$ es una mezcla libre de azufre y viscosidad elevada (2,8cSt), las cuales en proporciones de hasta $10 \%$ en volumen en el combustible condujeron a mezclas con bajos contenidos de azufre de hasta $12,5 \mathrm{ppm}$ sin ver afectada la calidad lubricante del mismo. Este aspecto es de gran interés puesto que a menudo este tipo de parámetros suelen ser contradictorios (caso de las emisiones de material particulado y óxidos de nitrógeno), donde la reducción en los niveles de azufre puede aumentar el riesgo de una lubricidad pobre e inadecuada del combustible, ya que durante los procesos de remoción de azufre, se retiran muchos de los hidrocarburos pesados responsables de la lubricidad natural del diésel [2].

Tabla 3. Propiedades estimadas de las mezclas combustibles con concentración de nanoaditivos de 0, 10 y $20 \mathrm{ppm}$.

\begin{tabular}{cccccc}
\hline Parámetro & Unidad & B4 & B4-10ppm & B4-20ppm & $\begin{array}{c}\text { Especificación } \\
\text { Res. 18 2087 de 2007 }\end{array}$ \\
\hline $\begin{array}{c}\text { Densidad a } 15^{\circ} \mathrm{C} \\
\text { Viscosidad cinemática } \\
\text { a 40 } \mathrm{C}\end{array}$ & $\mathrm{kg} / \mathrm{m}^{3}$ & 844,8 & 847,3 & 849,8 & ---- \\
Número de cetano & $\mathrm{cSt}$ & 2,5663 & 2,8961 & 3,2682 & $1,9-5,0$ \\
Oxígeno & \%masa & 0,0052 & 0,0109 & 0,0166 & Mínimo 43 \\
Azufre & \%masa & 0,0014 & 0,0013 & 0,0012 & Máximo 0,005 \\
\hline
\end{tabular}

Por otro lado, las mezclas combustibles B4-10ppm y B4-20ppm mostraron aumentos en el contenido de oxígeno, lo cual se podría traducir en una quema mucho más completa del combustible en los motores y reducciones en las emisiones de material particulado, pero sin despreciar sus posibles efectos en la formación de emisiones de óxidos nitrosos [16]. 


\section{Predicción de las emisiones de gases contaminantes y material particulado}

La predicción de los perfiles de emisiones de los gases contaminantes $\mathrm{CO}_{2}, \mathrm{NO}_{2}, \mathrm{SO}_{2}$ y material particulado para las mezclas combustibles con nanoaditivos se estimó usando el software DiéselRK. De igual forma, se evaluaron características importantes del funcionamiento del motor, tales como la temperatura máxima alcanzada dentro de los cilindros, el consumo especifico de combustible y el retraso en el tiempo de ignición. En las Figuras 1 y 2 , se observan las estimaciones encontradas para las emisiones de gases contaminantes y material particulado, y los resultados para las características en el motor. Estas predicciones se realizaron considerando una variación de la velocidad del motor en el rango de 20003000 rpm, para las distintas mezclas combustibles con nanoaditivos (0, 10 y 20ppm). La Tabla 4 presenta las especificaciones técnicas del motor seleccionado para la estimación en el software, el cual fue el asociado al auto Renault Logan 85hp $1,5 \mathrm{dCi}[17]$.

Tabla 4. Características técnicas del motor Renault Logan $85 \mathrm{hp} 1,5 \mathrm{dCi}$.

\begin{tabular}{cc}
\hline Renault Logan $\mathbf{8 5} \mathbf{~ h p ~} \mathbf{1 . 5} \mathbf{~ d C i}$ & Características del motor \\
\hline Motor tipo K9K 796 & Ocho válvulas \\
Capacidad $1461 \mathrm{cc}$ & Potencia máxima de 63 a $85 \mathrm{~kW} / \mathrm{hp}$ \\
Bore $76 \mathrm{~mm}$ & Radio de compresión de 17,9 \\
Stroke $80,5 \mathrm{~mm}$ & Cuatro cilindros \\
Velocidad motor a máx. potencia & $3750 \mathrm{rpm}$ \\
Sistema de inducción & Turbocompresor + Intercooler \\
Combustible & Diesel \\
\hline
\end{tabular}

La predicción de las emisiones de $\mathrm{CO}_{2}$ se muestra en la Figura 1(a), donde se observan disminuciones ligeras de este Gas Efecto Invernadero (GEI) de hasta $0,59 \%$ y $1,19 \%$ a $2000 \mathrm{rpm}$ para las mezclas combustibles B4-10ppm y B4-20ppm, respectivamente.

Los resultados mostrados reflejan mejoras en las emisiones de $\mathrm{CO}_{2}$ comparado con lo reportado por Keskin y colaboradores quienes usaron aditivos a base de dióxido de manganeso $\left(\mathrm{MnO}_{2}\right)$ y óxido de magnesio (MgO) en diésel, el cual les ocasionó incrementos en las emisiones de $\mathrm{CO}_{2}$ de hasta un $2,9 \%$ con revoluciones de motor de $2000 \mathrm{rpm}$ [18]. Las emisiones de dióxido de azufre $\mathrm{SO}_{2}$ estimadas para las mezclas de combustibles con nanopartículas se muestran en la Figura 1(b), en la cual se observan disminuciones promedio de $5,09 \%$ y $10,9 \%$ para las mezclas B4-10ppm y B4$20 \mathrm{ppm}$, respectivamente, para todo el rango de velocidad de motor estudiadas, sugiriendo que la generación de estos gases depende principalmente del contenido de azufre del combustible. Estos resultados corroboran los valores estimados para el contenido de azufre de las mezclas combustibles con nanopartículas, en los cuales se encontraron bajos contenidos de azufre de hasta 5,28\% y
$10,53 \%$ para las mezclas B4-10ppm y B4-20ppm, respectivamente.

El empleo de la suspensión base ácido oleiconanopartículas, libre de azufre, en formulaciones de hasta $10 \%$ en volumen es responsable de la reducción del azufre contenido inicialmente en el combustible fósil, y de este modo de las menores emisiones de óxidos de azufre durante la combustión del mismo. Esto representa un impacto directo sobre la calidad del aire, y favorece el uso de sistemas postcombustión disminuyendo los riesgos de taponamiento, corrosión, entre otros. $[1,6]$.

En relación a los óxidos nitrosos, los resultados revelaron un incremento desde $2,1 \mathrm{~g} / \mathrm{kWh}$ hasta $3,1 \mathrm{~g} / \mathrm{kWh}$ a $2000 \mathrm{rpm}$ con la adición de las nanopartículas a la mezcla combustible en la concentración máxima de 20ppm. Esto estaría relacionado con el aumento en la temperatura promedio alcanzada en los cilindros (Figura 2), además del mayor contenido de oxígeno en las mezclas combustibles producto de la adición de ácido oleico durante la preparación del nanoaditivo [4]. Esto concuerda con lo reportado por Mehta et al., quienes indicaron aumentos en las emisiones de óxidos de nitrógeno $\mathrm{NO}_{\mathrm{x}}$ con la adicción de nanopartículas de aluminio en diésel [19]. 

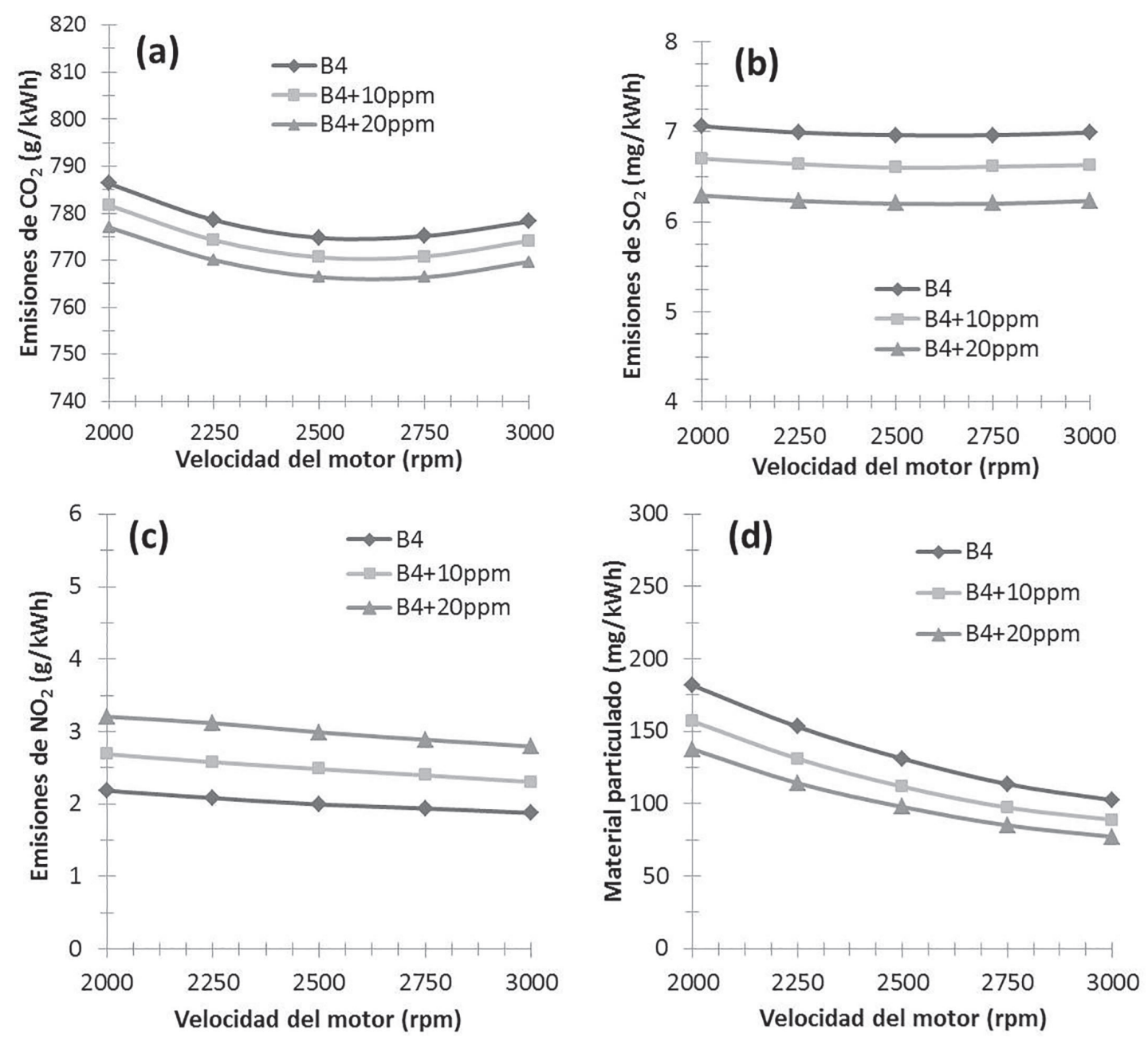

Figura 1. Predicción de emisiones para las mezclas combustibles como función de la concentración de nanoaditivos basados en ácido oleico y alúmina. (a) Emisión de $\mathrm{CO}_{2}$, (b) $\mathrm{SO}_{2}$, (c) $\mathrm{NO}_{2}$ y (d) materia.

El aumento en las emisiones de óxidos nitrosos reitera la necesidad del empleo de surfactantes que conduzcan a suspensiones de mayores cantidades de aditivos, reduciendo de este modo la relación ácido oleico-nanopartículas, y a su vez, los contenidos de oxígeno en la mezcla. Lo anterior asegurando que la formulación de este surfactante en la suspensión no deteriore las características actuales del combustible como viscosidad, número de cetano y emisiones de material particulado, además de las mejoras logradas en emisiones de gases como $\mathrm{CO}_{2}$ y $\mathrm{SO}_{2}$ [18].

La Figura 1(d) presenta las emisiones de material particulado para las mezclas combustibles con nanoaditivos. En esta figura se puede observar una tendencia en la reducción del material particulado con el aumento en la concentración del nanoaditivo a lo largo del intervalo (2000-
$3000 \mathrm{rpm})$, con reducciones hasta del $14,7 \%$ y $25,4 \%$ a $2500 \mathrm{rpm}$ para las mezclas B4-10ppm y B4-20ppm, respectivamente. Las reducciones en las emisiones se deben a los elevados contenidos de oxígeno presentes en las mezclas, lo cual condicionó la relación de oxígeno-combustible favorable dentro del cilindro, resultando en combustiones mucho más completas, además del bajo contenido de azufre en las mezclas, puesto a que estas también contribuyen a la generación de partículas finas en forma de sulfatos. Lo anterior concuerda con los resultados reportados por Bello y colaboradores, los cuales reportan reducciones de hasta $27 \%$ en emisiones de material particulado con la reducción controlada del contenido de azufre del combustible [20]. Estos resultados son favorables comparados con los reportados por Basha y Gopal, quienes presentan reducciones 
máximas del $15 \%$ y $18 \%$ para nanopartículas de alúmina en biodiésel en concentraciones de 50 y $100 \mathrm{ppm}$, respectivamente [2].

En la Figura 2 (a) se puede observar que la temperatura del cilindro presentó aumentos con la adición de las nanopartículas de alúmina, observándose incrementos de hasta $6,8^{\circ} \mathrm{C}$ y $13,2^{\circ} \mathrm{C}$ a 2250rpm para las mezclas B4-10ppm y B4-20ppm, respectivamente. La temperatura alcanzada dentro de los cilindros está estrechamente relacionada con la capacidad calorífica del combustible y la calidad de la combustión, favoreciendo la quema de residuos en el interior del mismo y reduciendo los riegos de incrustaciones de carbón en las válvulas y pistones.
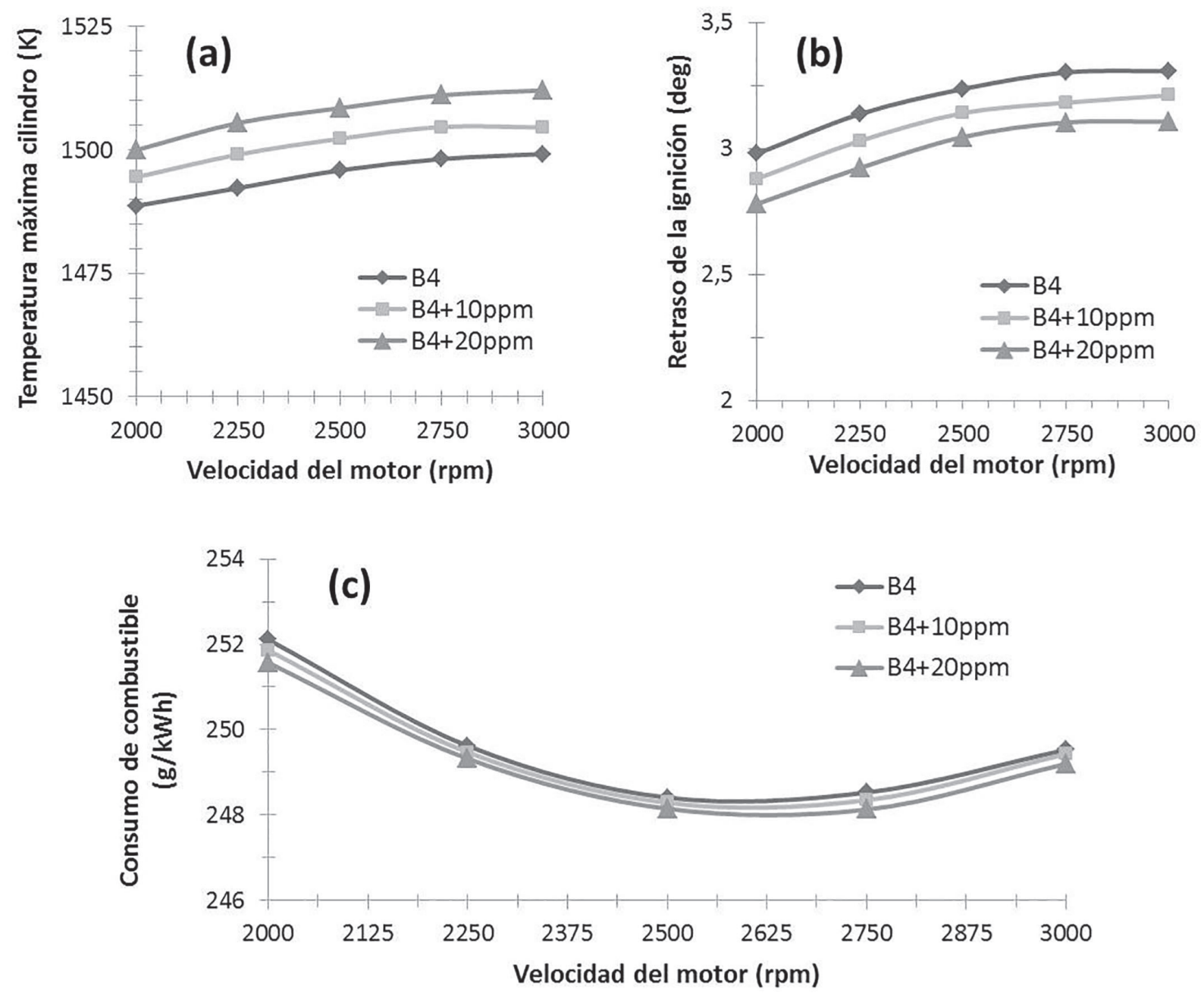

Figura 2. Parámetros de funcionamiento del motor usando las mezclas combustibles con nanoditivos.

(a) Temperatura máxima del cilindro; (b) Retraso de la ignición; y (c) Consumo de combustible. 
En la Figura 2(b) se presentan la relación entre el retardo de ignición y la composición de las mezclas combustibles. Se pudo observar que las mezclas modificadas con nanopartículas de alúmina exhiben retardos de ignición mucho más bajos en comparación con la mezcla B4 sin aditivo; encontrándose reducciones máximas de hasta $3,63 \%$ a $2750 \mathrm{rpm}$ y $6,81 \%$ a $2250 \mathrm{rpm}$ para las mezclas B4-10ppm y B4-20ppm, respectivamente. Esto evidenció la capacidad de autoignición superior de estas mezclas, lo cual puede deberse al aumento en los números de cetano tras el empleo del aditivo (aproximadamente dos unidades). En la Figura 2(c) se muestra el consumo específico para las mezclas combustibles. Este parámetro no presentó mayores cambios con la adición de nanopartículas, observándose reducciones máximas de $0,1 \%$ y $0,21 \%$ a $2000 \mathrm{rpm}$ para las mezclas B4-10ppm y B420ppm, respectivamente. Estas reducciones son poco significativas comparados con lo reportado por Keskin et al. [18], quienes indicaron porcentajes de hasta $4,16 \%$ y $2,02 \%$ a $1800 \mathrm{rpm}$ para aditivos basados en manganeso y magnesio en diésel, respectivamente.

Dado que el encendido de los combustibles se produce solo por efecto de presión en los motores diésel, el número de cetano en las mezclas es sinónimo de calidad del mismo, por lo que modificaciones que conduzcan a reducciones en este parámetro no serían las más adecuadas. Una combustión con calidad está representada en una ignición rápida acompañada de un quemado total y homogéneo del combustible. Estas reducciones en los tiempos de ignición revelan que el aumento en la viscosidad del combustible producto de la adición de las nanopartículas no generó ningún tipo de inconveniente durante la atomización y el mezclado del mismo dentro de los cilindros, el cual es una de las grandes preocupaciones del empleo de mezclas con niveles relativamente altos de lubricidad. Otra situación particular, fue la reducción en la capacidad calorífica de las mezclas modificadas, la cual pudo facilitar las condiciones de temperatura para la autoignición en un menor tiempo.

Las mezclas combustibles formuladas en el presente trabajo mostraron mejoras en emisiones de $\mathrm{CO}_{2}$, $\mathrm{SO}_{2}$ y material particulado, producto de los altos contenidos de oxígeno conseguidos con el empleo del aditivo, lo anterior sin reducir la capacidad de autoignición del mismo; sin embargo, esto condujo a aumentos desfavorables en las emisiones de $\mathrm{NO}_{\mathrm{x}}$. Sin embargo, el bajo contenido de azufre de estas mezclas facilita el empleo de sistemas de control de emisiones con el fin de compensar estos aumentos en dichas emisiones. Lo anterior sugiere la preparación de mezclas combustibles favorable para su uso en motores, ajustándose más fácilmente a exigencias ambientales y de consumo.

\section{Conclusiones}

La predicción de las emisiones de gases contaminantes y material particulado mostraron reducciones mínimas en las emisiones de gases $\mathrm{CO}_{2}$, por debajo de $1,2 \%$ en comparación con el combustible B4 libre de aditivos; en cuanto a las emisiones de $\mathrm{SO}_{2}$, se reportaron mejoras importantes de hasta $10,92 \%$ a $2500 \mathrm{rpm}$ para la mezcla B4-20ppm, acompañado de incrementos sustanciales de hasta $28,1 \%$ en la viscosidad. Lo cual sugiere la presencia de mezclas combustibles libres de azufre, sin reducciones significativas en su calidad lubricante.

Las emisiones de material particulado revelaron reducciones significativas de hasta $14,7 \%$ a 2500 rpm y $25,4 \%$ a 2500 rpm para las mezclas B410 ppm y B4-20ppm, producto de los contenidos mayores en oxígeno de las mezclas modificadas (por encima del doble en comparación al B4) y el aumento de las temperaturas alcanzadas dentro de los cilindros. Sin embargo, el aumento en estos parámetros condujo a mejores condiciones para la formación de $\mathrm{NO}_{\mathrm{x}}$, los cuales mostraron incrementos de hasta $1 \mathrm{~g} / \mathrm{kWh}$ a $2500 \mathrm{rpm}$ para las mezclas B4-20ppm. Esto refleja la necesidad de una óptima relación nanopartículas-ácido oleico, de tal modo que la mayor contribución en la mejora de propiedades sea por parte del nanoaditivo evitando el incremento de emisiones de $\mathrm{NO}_{2}$ producto del aumento en los contenidos de oxígeno que implica el uso de cantidades elevadas de ácido oleico en las mezclas combustibles.

Por último, se observaron que las mezclas modificadas con nanopartículas de alúmina exhiben retardos de ignición mucho más bajos a la mezcla B4 sin aditivo, encontrándose reducciones máximas de hasta 6,81\% para la mezcla B4-20ppm. Esto evidencia la capacidad de auto ignición superior de estas mezclas, debido al aumento en los números de cetano tras el empleo del aditivo. Así, el uso de las nanopartículas en las mezclas combustibles condujo a mejoras significativas en propiedades claves para el funcionamiento adecuado de motores y reducciones en emisiones de $\mathrm{CO}_{2}, \mathrm{SO}_{2}$ y material particulado, sin embargo, 
sus altos contenidos de oxígeno condujeron a aumentos desfavorables en las emisiones de óxidos nitrosos; no obstante el bajo contenido de azufre de la mezclas formuladas posibilita el empleo de sistemas de control de gases (EGR, filtros, catalizador), sin limitaciones por el deterioro en su funcionamiento, con el cual podría retribuir esta limitante, y conseguir ajustarse fácilmente dentro de requerimientos ambientales y de consumo. Asimismo, no se descarta la posibilidad de obtener mejoras aún más relevantes al aumentar la dosis de nanoaditivos con la formulación de relaciones óptimas entre nanopartículas y ácido oleico con el empleo de agentes surfactantes apropiados.

En adición, se pudo observar que las propiedades fisicoquímicas determinadas (punto de inflamación y viscosidad) se hallan dentro de los requisitos de calidad del combustible diésel y sus mezclas con biocombustibles para su uso en motores (Resolución 182087 de 17 de diciembre de 2007), lo cual sugiere la susceptibilidad de este tipo de suspensiones de nanopartículas en la mejora de características claves de combustibles.

\section{Agradecimientos}

Los autores del artículo desean agradecer a la Universidad de Cartagena por el apoyo financiero para el desarrollo de esta investigación (Plan Fortalecimiento Grupos de Investigación Actas No. 012-2015 y 038-2015).

\section{Referencias bibliográficas}

[1] Sajith V, Sobhan CB, Peterson GP. Experimental investigations on the effects of cerium oxide nanoparticle fuel additives on biodiesel. Advances in Mechanical Engineering 2010;2:1-6.

[2] Basha SA., Gopal KR. A review of the effects of catalyst and additive on biodiesel production, performance, combustion and emission characteristics. Renewable and Sustainable Energy Reviews 2012;16(1):711-7.

[3] Yong-Qiang R, Zhi-Ning H, Yan F. Evaluation on combustion properties of nanoparticle as fuel additive. Advanced Materials Research 2011;335(336):1516-9.

[4] Jones $\mathrm{M}, \mathrm{Li} \mathrm{CH}$, Afjeh A. Peterson G. Experimental study of combustion characteristics of nanoscale metal and metal oxide additives in biofuel (ethanol). Nanoscale Research Letters 2011;6(246):1-12.
[5] Selvan M, Anand R, Udayakumar M. Effects of cerium oxide nanoparticle addition in diesel and diesel-biodiesel-ethanol blends on the performance and emission characteristics of a $\mathrm{Cl}$ engine. ARPN Journal of Engineering and Applied Sciences 2009;4(7):1-6.

[6] Ganesh D, Gowrishankar G. Effect of Nanofuel additive on emission reduction in a Biodiesel fuelled $\mathrm{Cl}$ engine. IEEE International Conference in Electrical and Control Engineering 2011;3453-9.

[7] Elias M, Mahbubul I, Saidur R, Sohel M, Shahrul M, Khaleduzzaman S, Sadeghipour $\mathrm{S}$. Experimental investigation on the thermophysical properties of $\mathrm{Al}_{2} \mathrm{O}_{3}$ nanoparticles suspended in car radiator coolant. International Communications in Heat and Mass Transfer 2014;:54:48-53.

[8] Sundar L, Sharma K, Naik M, Singh K. Empirical and theorical correlations on viscosity of nanofluids: A review. Renewable and Sustainable Energy Reviews 2013;25:67086.

[9] Chandrasekar M, Suresh S, Bose A. Experimental investigations and theoretical determination of thermal conductivity and viscosity of $\mathrm{Al}_{2} \mathrm{O}_{3} /$ water nanofluid. Experimental Thermal and Fluid Science 2010;34:210-6.

[10] Dadgostar N, Shaw J. A predictive correlation for the constant-pressure specific heat capacity of pure and ill-defined liquid hydrocarbons. Fluid Phase Equilibria 2011;313:211-26.

[11] Sivaramakrishnan K, Ammal A, Ravikumar P. Determination of higher heating value of biodiesels. International Journal of Engineering Science and Technology (IJEST) 2011;3(11):7981-7.

[12] Parhizgar H, Reza M, Eftekhari A. Modeling of vaporization enthalpies of petroleum fractions and pure hydrocarbons using genetic programming. Journal of Petroleum Science and Engineering 2013,112:97-104.

[13] Peñaloza A, Rincón A. Determinación del efecto del uso de nanopartículas de alúmina en mezcla diésel/biodiésel para mejora de propiedades fisicoquimicas y reducción de emisiones (Tesis Ingeniería Química) Cartagena, Colombia: Universidad de Cartagena; 2014.

[14] Tanvir S, Quiao L. Surface tension of Nanofluidtype fuels containing suspended nanomaterials. Nanoscale Research Letters 2012;7(226):1-10.

[15] Benjumea P, Agudelo J, Agudelo A. Basic properties of palm oil biodiesel-diesel blends. Fuel 2008, 87:2069-75. 
[16]Rodriguez, J. Estimación de propiedades termodinámicas. En: Modelado, simulación y optimización de procesos químicos. Scenna N, Editor. Argentina: Editorial Edutecne; 1999, p. 303-27.

[17]Patil AR, Taji SG. Effect of oxigenated fuel additive on diesel engine performance an emission:AReview. IOSR Journal ofMechanical and Civil Engineering 2013;2320(334):30-5.

[18] Keskin A, Gürü M, Altıparmak D. Influence of metallic based fuel additives on performance and exhaust emissions of diesel engine. Energy Conversion and Management. 2011;52(1):605.

[19] Mehta R, Chakraborty M, Parikh P. Nanofuel: Combustion, engine performance and emissions. Fuel 2014;120:91-7.

[20]Bello A, Torres J, Herrera J, Sarmiento J. Effect of diesel properties on the emissions of particulate matter. CT\&F. 2000;2(1):31-46. 